\title{
In-Flight Validation of a Robust Flight Controller Featuring Anti-Windup Compensation
}

\author{
Christian Weiser * and Daniel Ossmann ${ }^{\dagger}$ \\ Institute of System Dynamics \& Control \\ German Aerospace Center (DLR), 82234 Wessling, Germany \\ Matthias Heller $\ddagger$ \\ Institute of Flight System Dynamics \\ Technical University of Munich (TUM), 85748 Garching, Germany
}

\begin{abstract}
In-flight demonstration and verification of a robust flight control design including an advanced anti-windup strategy is presented in this paper. The test aircraft, an Ultrastick 120 unmanned aircraft, is equipped with a pitch- and roll-attitude command flight-control system for remotely controlled augmented flight. The longitudinal aircraft dynamics are enhanced with an $H_{\infty}$-based control design. The controller additionally comprises an advanced anti-windup strategy to avoid integrator windup during actuator saturation. The lateral axis features a linear-parameter varying controller which provides robust performance over the whole velocity range. The controllers as well as the anti-windup algorithm are verified and validated in a non-linear simulation environment and by various flight tests. The paper provides detailed insight in the design process of the controllers, as well as a comprehensive discussion on the simulation and flight test results.
\end{abstract}

\section{Introduction}

In this paper an advanced flight control algorithm for a small unmanned vehicle, the Ultrastick 120 (displayed in Figure 1), is developed. Robust control techniques are employed to derive longitudinal and lateral control laws, which provide the external pilot with satisfactory handling qualities and allow an automated flight for predefined command input signals. The design of basic flight control laws for aircraft is usually straightforward and common control architectures are provided in literature, see, e.g., Ref. 1 for a comprehensive overview. Longitudinal and lateral augmentation is achieved with simple proportional-integral-derivative controllers. Nowadays active reduction of aeroservoelastic effects ${ }^{2-4}$ resulting from a coupling of the structural dynamics of the airframe with the aerodynamics, as well as active load alleviation capabilities $^{5,6}$ shall be considered during the flight control system design. To be able to implement these advanced functionalities, additional measurements in the form of load sensors on the wings and additional control inputs as actuators are integrated on the aircraft. However, the resulting multiple-input multiple-output (MIMO) system cannot be tackled easily with classical control design methods. Robust control techniques are sophisticated methods which allow the control design for such MIMO systems in a straightforward way.

While the Ultrastick 120 does not have any significant aeroelastic effects nor requires active load reduction, the specific motivation herein is to perform a complete model-based robust control law design process, i.e. from modeling to flight-test. As the experience of actually implementing and flying $H_{\infty}$ and linear parametervarying (LPV) controllers is limited, the Ultrastick 120 is utilized as a small-scale and low cost test bed to gather experience with these advanced control design techniques. This also includes the tackling of all arising practical issues, which may also emerge when developing robust controllers for larger and more advanced aircraft systems. Thus, this paper explicitly overcomes issues such as integrator-windup as well as the in-flight

*Research Assistant: christian.weiser@dlr.de

${ }^{\dagger}$ Research Scientist: daniel.ossmann@dlr.de, AIAA Member

${ }^{\ddagger}$ Rudolf Diesel Fellow of TUM-IAS, Professor @ TUM: matthias.heller@tum.de 


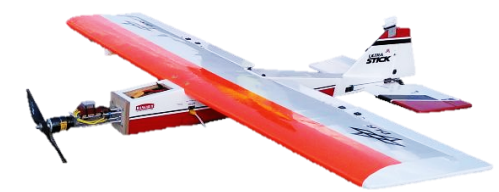

Figure 1. The DLR Ultrastick 120 aircraft.

activation of the control laws. This idea of using the Ultrastick 120 as testbed has similarly been utilized in various research and technology projects. For example in Ref. 7 a detailed description of the aircraft and different applications are presented, one being the implementation of a standard baseline control law and an auto-pilot using classical proportional and proportional-integral feedbacks, including a simple anti-windup logic for the integrators. Further, in Refs. 8,9 the Ultrastick 120 aircraft is used to test a backstepping controller autopilot.

For the design of the controllers in this paper, an available non-linear model of the Ultrastick 120 is linearized with respect to different flight speeds to facilitate the model based design procedure in section II. A generic weighting structure for the robust control design for both, the lateral and longitudinal axis is derived. While for the longitudinal controller a classical $H_{\infty}$ design is applied, the lateral axis is augmented with a linear parameter-varying controller to provide similar flying qualities and robustness over the whole velocity envelope. The longitudinal controller is additionally augmented with an anti-windup strategy, presented in section III to avoid integrator windup in case of saturation in control surface deflection. The designed flight control law is extensively tested in section IV using a non-linear model of the aircraft, including aerodynamics, actuators and sensor dynamics. The testing also involves, besides maneuvers in all axes for different flight speeds, extensive tests of the anti-windup strategy and activating the controller in flight to gain confidence for the flight test. The latter is carefully evaluated before flight-testing using simulations to avoid unexpected aircraft behavior. The results from an extensive flight test campaign are presented in section V. For comparison with simulation results, one part of the flight tests was conducted automatically, i.e. the input signal was predefined and commanded by the flight control computer. A second set of closed loop tests was flown in augmented flight with the external pilot in the control loop. To verify the anti-windup strategy, additional tests with reduced elevator saturation limits to trigger the anti-windup algorithm where conducted and are discussed in section V.

\section{Controller Design}

For both, the lateral and longitudinal axis a controller which allows to command and track the attitude angles, i.e. pitch attitude in the longitudinal axis and roll attitude in the lateral axis, shall be designed. This enables a pilot to fly the unmanned aircraft in the most intuitive way. The design goal in both axes is a fast tracking behavior with the complimentary goal of no or only slight overshoots. In practice the design is limited by the actuator bandwidths, together with limited available actuator position range. Moreover, the actuation effort shall be minimized, which will be implemented via weighting functions in the design process. As basis for the controller design a six degree-of-freedom (DOF) non-linear dynamic model of the Ultrastick 120 aircraft is available. The basic geometric data of the aircraft is displayed in Table 1, aerodynamic data is available through lookup tables and was obtained from extensive wind tunnel testing. ${ }^{10,11}$ By trimming for steady level flight with flight path angle $\gamma$ equal zero over the full velocity envelope from 15 to $35 \mathrm{~m} / \mathrm{s}$, a set of trim points is obtained. To facilitate a model based control design, the non-linear model is linearized at these trim points providing a set of linear time-invariant (LTI) models which are describing the aircraft dynamics. To design $H_{\infty}$ control laws, only one trim point of the envelope is used to design a robust controller, which achieves adequate performance for the aircraft response over the whole envelope. In the lateral axis, however, the design is extended to a LPV controller. This is necessary to provide satisfactory system responses for the whole velocity grid, which could not be achieved with a constant controller. The LPV controller is scheduled with the velocity measurement. Additionally, for the pitch axis an anti-windup strategy is implemented, which turned out to be necessary at low speeds to avoid integrator windups.

For all controllers a two DOF structure is chosen, separating the feedback from the command path, which allows to enhance the performance in terms of faster responses to changes in reference signal without 
Table 1. Basic parameters of the DLR Ultrastick 120 aircraft.

\begin{tabular}{lcc} 
Parameter & Unit & Value \\
\hline Mass & $m$ & $8.13 \mathrm{~kg}$ \\
Length & $l$ & $1.26 \mathrm{~m}$ \\
Span & $b$ & $1.92 \mathrm{~m}$ \\
Chord & $\bar{c}$ & $0.433 \mathrm{~m}$ \\
Wing Surface & $S$ & $0.769 \mathrm{~m}^{2}$
\end{tabular}

compromising stability. The control problem for both controllers is formulated as closed loop interconnection shown in Figure 2, based on considerations provided, discussed and applied for example in Refs. 12-15. In the generalized plant of Figure 2 the inputs are the reference signal $r$, the input disturbance $d_{1}$, and the output disturbance $d_{2}$. The outputs are the weighted tracking error $e=r\left[\begin{array}{ll}1 & 0\end{array}\right]-y$, as there is always one reference signal and multiple measurement signals, the weighted control effort $u$, and if necessary, any nonmeasured weighted performance outputs $p$. This generic structure is applied for the design in longitudinal and also lateral axis, where only for the lateral controller the additional performance output $p$ is used. The resulting input-output map for the control design is given by

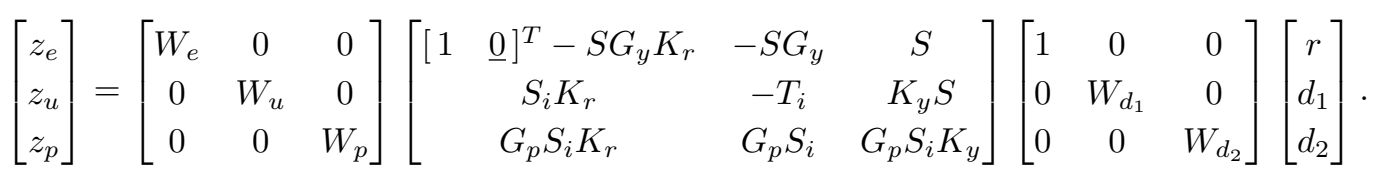

In (1) $K_{y}$ is the feedback part and $K_{r}$ the feed-forward part of the controller, $G_{y}$ is the transfer matrix from command inputs to measurable outputs, and $G_{p}$ is the transfer matrix from the command inputs to the (non-measurable) performance outputs. Further, $S=\left(I+G_{y} K_{y}\right)^{-1}$ is the sensitivity function, and $S_{i}=\left(I+K_{y} G_{y}\right)^{-1}$ and $T=K_{y} G_{y}\left(I+K G_{y}\right)^{-1}$ are the input sensitivity and input complementary sensitivity function, respectively $T_{r}=K_{r} G S$ complimentary sensitivity on reference signal. $W_{e}, W_{u}$, and $W_{p}$ are the diagonal output weighting matrices, and $W_{d_{1}}$ and $W_{d_{2}}$ are the diagonal input weighting matrices to be chosen in the design process. Note that the aircraft models $G$ are scaled as proposed in Ref. 12 by input-output scalings. As indicated in Figure 2, only $W_{e}$ and $W_{u}$ are selected as dynamic filters, while all other filters are constant. The entries for the shaping filter $W_{u}$ are selected as first-order transfer functions with unit gain up to the available bandwidths $\omega_{a, i}$ for $i=1, \ldots, n$ of each control input and approximately differentiating behavior beyond that frequency. In $W_{e}$ only the first element is dynamic in order to weight the tracking behavior, while all other channels are set to constant values to weight the outputs. As in this paper integral behavior is demanded in the tracking channel, the choice for the first entry is a first-order transfer function with approximately integral behavior up to the desired bandwidth $\omega_{b}$, which reduces the sensitivity up to this selected bandwidth.

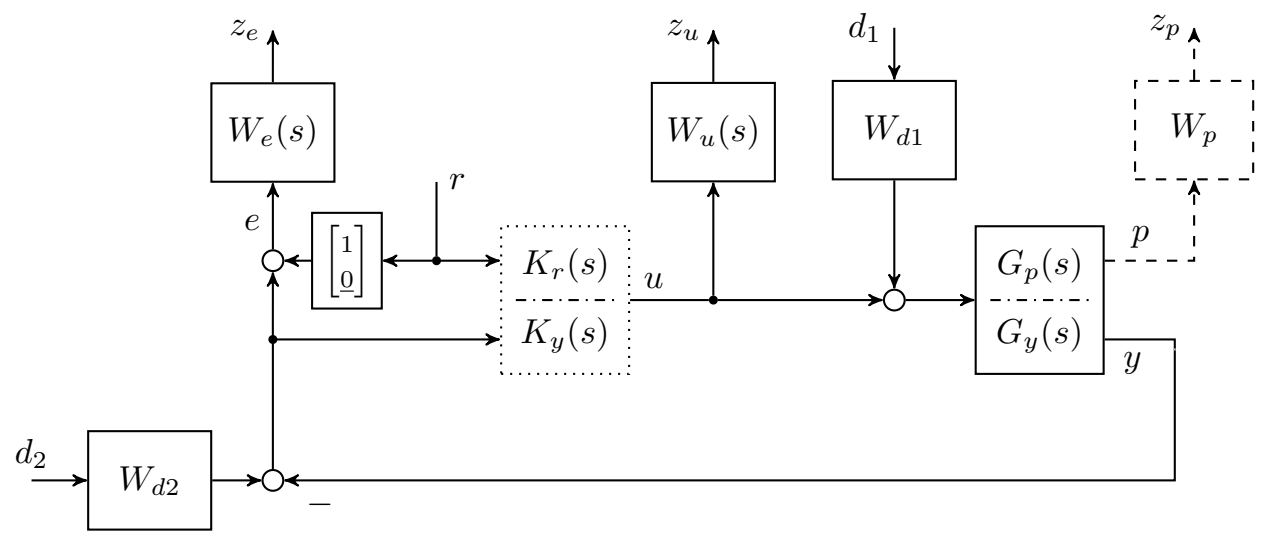

Figure 2. Generic $H_{\infty}$ weighting structure used for the longitudinal and lateral controller design. 
The weighting filters are selected so that the general design goals for pitch and roll attitude control, namely rise times smaller than one second and no overshoot in the commanded signals, are achieved. Further, the controllers shall meet common requirements for gain and phase margins (6 dB, $45 \mathrm{deg})$. Reference signals are pitch attitude command in longitudinal and roll attitude command in lateral axis control.

\section{A. Longitudinal Controller}

The LTI design model for the longitudinal dynamics is a single-input multiple-output (SIMO) system with elevator deflection as control input $(u=\eta)$, pitch attitude $\Theta$ and pitch rate $q$ as measurable outputs stacked together in the output vector $y$. For the controller synthesis the weighting structure from Figure 2 is used, where the available control bandwidth limitation is set to $\omega_{a, 1}=7.5 \mathrm{rad} / \mathrm{s}$ and the desired bandwidth for sensitivity reduction in $W_{e}$ is set to $\omega_{b}=5 \mathrm{rad} / \mathrm{s}$. The design results in a $4 \times 4$ LTI model to design the controller $K=\left[\begin{array}{ll}K_{r} & K_{y}\end{array}\right]^{T}$, where $K_{r}$ is the feed-forward controller and $K_{y}$ is the feedback control part. The pitch attitude is added to the structure as reference input $\left(r=\Theta_{\text {cmd }}\right)$. Note that in case of the longitudinal axis, the performance channel is not present, i.e. $G_{p}=0$. The LTI model $G$ itself is gained through linearization of the non-linear simulation model, trimmed for horizontal flight $(\gamma=0)$ at an airspeed of $34 \mathrm{~m} / \mathrm{s}$. This speed is at the upper end of the aircraft envelope and has been chosen for design, as the result of testing design approaches at different trim points. The outcome of design iterations showed at the high speed trim point best robustness indicators as gain and phase margins are achieved over the full envelope. In contrast, controller designs at medium or low speeds show higher deficiencies in either robustness parameters or tracking performance when assessed off the design points.

In Figure 3 the resulting closed loop magnitude plots of the sensitivity, the complementary sensitivity and the reference transmission functions are depicted. The sensitivity $(-)$ in the pitch attitude channel is reduced up to a frequency of around $3 \mathrm{rad} / \mathrm{s}$ providing good tracking behavior up to this frequency and respecting the limitation of the actuator bandwidth. In the pitch rate channel, the sensitivity is mainly reduced around the short period frequency $(\approx 10 \mathrm{rad} / \mathrm{s})$. Thus, this channel corresponds to a classical flight control design, where pitch dampers are employed to increase the damping in the short period mode. The reference transmission, from commanded pitch attitude to the pitch attitude of the aircraft, is reduced compared to the pure inner loop of the closed loop. As a higher bandwidth is favorable in the inner loop to provide enough damping and stability margins, the bandwidth is reduced in the feed-forward. This ensures that the command path still provides satisfactory response times to the pilot without being too aggressive. This is also confirmed by the step responses in pitch attitude and pitch rate in Figure 4 for different aircraft velocities. The controller also provides comparable responses over the whole velocity grid. The maximum pitch rate increases with higher trim velocity, however, staying in satisfactory range. For all speeds, the time domain criteria of an aperiodic response with a rise-time smaller than one second is fulfilled. For stability analysis, single loop cut stability margins at in- and outputs are calculated. For this, a linear, first order pitch actuator model with a time-constant of $0.07 \mathrm{~s}$ and linear, first order sensor models with time constants of $0.05 \mathrm{~s}$ are considered. The minimum phase margin over all velocities and channels is $47 \mathrm{deg}$, the minimum gain margin of about $9 \mathrm{~dB}$, indicating satisfactory robustness margins.

\section{B. Lateral Controller}

For the design of the lateral controller, a similar weighting scheme as presented for the longitudinal control design is applied. The goal is to track the roll attitude reference command. The measured output variables for the lateral controller are roll attitude, roll rate and yaw rate, i.e. $y=\left[\begin{array}{lll}\Phi & p & r\end{array}\right]^{T}$ ). Aileron and rudder $\left(u=\left[\begin{array}{ll}\xi & \zeta\end{array}\right]^{T}\right)$ are available as control inputs. The additional design freedom, arising from the second control input is used to reduce the lateral acceleration during turns, i.e. $a_{y}=0$. As lateral acceleration showed large uncertainties in comparisons between linear, non linear model and actual sensor data, the decision was made to use a model based approach with an additional performance output $p=a_{y}$ with the corresponding performance weight $W_{p}$ (dashed in Figure 2). This trades the exact tracking of $a_{y}=0$ for a strong improvement in robustness with still good reduction of lateral acceleration compared to PID controlled simulations. Note that the Ultrastick 120 is not equipped with side-slip angle measurement; therefore lateral acceleration is preferred to design a controller which ensures coordinated turning. For the controller synthesis the weighting structure from Figure 2 is used, where the available control bandwidth limitation is set to $\omega_{a, 1}=\omega_{a, 2}=7.5 \mathrm{rad} / \mathrm{s}$ as the actuators for ailerons and rudder are identical. The desired bandwidth for sensitivity reduction in $W_{e}$ is set to $\omega_{b}=5 \mathrm{rad} / \mathrm{s}$. A LPV controller design ${ }^{16}$ with the measured 

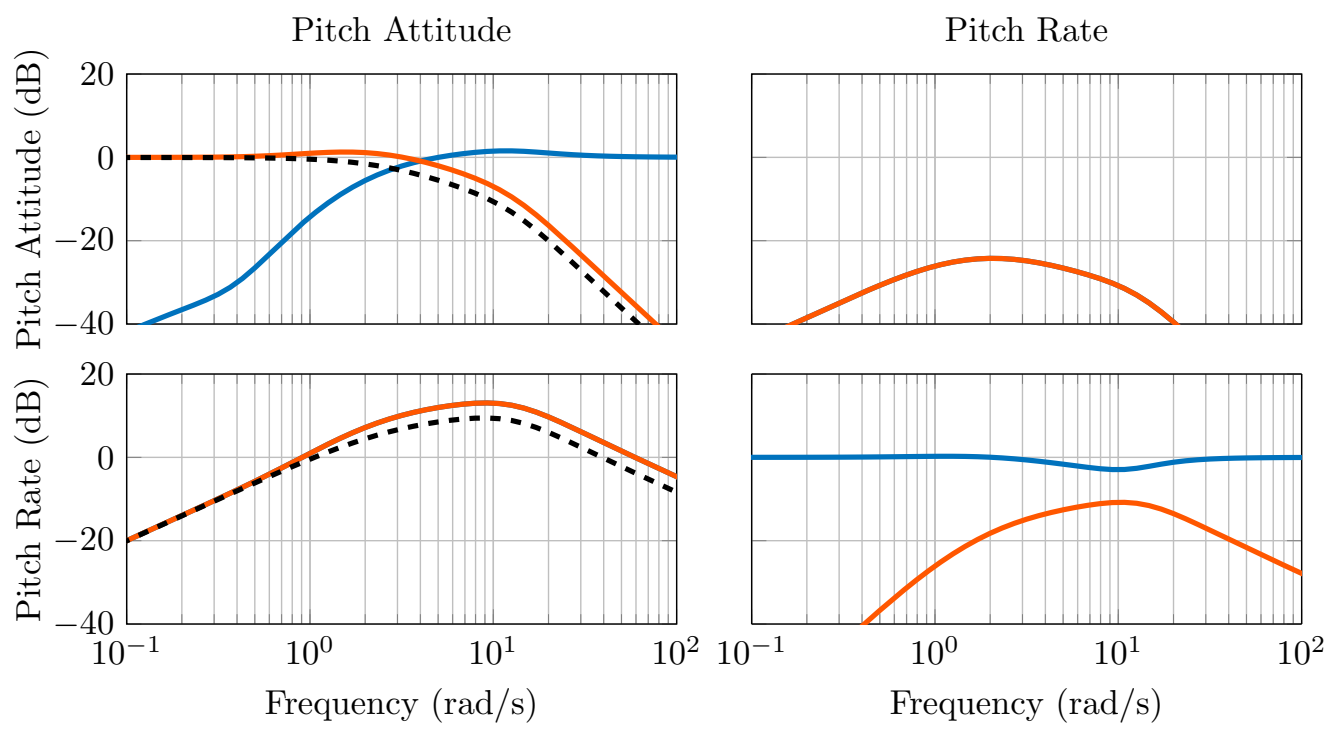

Figure 3. Bode plots of sensitivity $(-)$, complimentary sensitivity $(-)$, and reference transmission $(---)$ at design point of the $H_{\infty}$ controller.
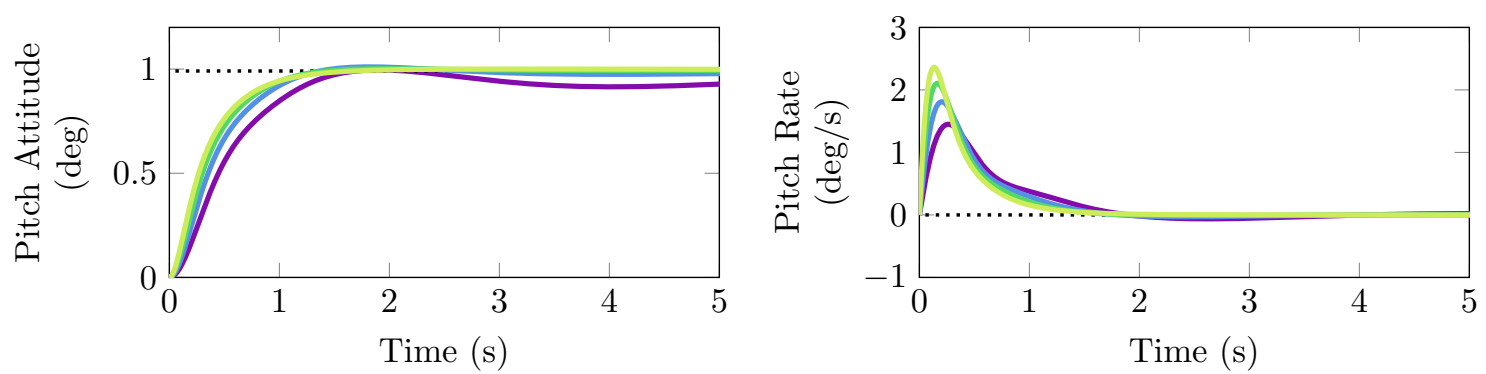

Figure 4. Pitch attitude and pitch rate step responses to a pitch attitude step command at $18 \mathrm{~m} / \mathrm{s}(\longrightarrow)$, $24 \mathrm{~m} / \mathrm{s}(\square), 30 \mathrm{~m} / \mathrm{s}(\square), 36 \mathrm{~m} / \mathrm{s}(-)$.

velocity as scheduling parameter is designed in the lateral axis. By the scheduling of the controller robust performance over the whole velocity range is achieved. A set of LTI models for the lateral dynamics over the velocity grid $V=\{13,15,17,20,25,30,35\} \mathrm{m} / \mathrm{s}$ is used for the design. For the LPV design process the algorithms implemented in Ref. 17 are used.

In the left diagram of Figure 5 the Bode magnitude plot of the sensitivity, complementary sensitivity and reference transmission of the roll attitude channel is depicted for the minimum, maximum, and mean flight speed. The sensitivity is reduced in the worst case to $1.5 \mathrm{rad} / \mathrm{s}$, providing adequate tracking behavior below this frequency. Again, the feed-forward path is used to reduce the bandwidth in the reference transmission a bit compared to the complementary sensitivity function, i.e. in the inner loop. Clearly, the feed-forward path is not extensively used in this design, as the complementary sensitivity and the reference transmission closely match each other. The right diagram shows a singular value plot from the rudder and aileron inputs to the lateral acceleration, i.e. the additional performance output. Clearly visible is the reduction of the peaks in the closed loop (-) compared to the open loop (-). Thus, additional damping is added by the controller to reduce the lateral acceleration during a roll attitude command.

Phase and gain channels are determined using the open loop responses for all channels. The worst case phase margin for the LPV design is around $45 \mathrm{deg}$ and the worst case gain margin is $16 \mathrm{~dB}$ when again taking linear actuator and sensor models into account. The resulting margins indicate excellent robustness against gain perturbations and still satisfactory margins against phase perturbations. Note that an $H_{\infty}$ design first tested for the lateral axis resulted in much poorer margins. The step responses to a roll attitude command in Figure 6 show satisfactory rise times and comparable responses over the whole velocity grid. Moreover, only small overshoots are encountered in the roll and yaw rate responses. As mentioned in above, the controller 

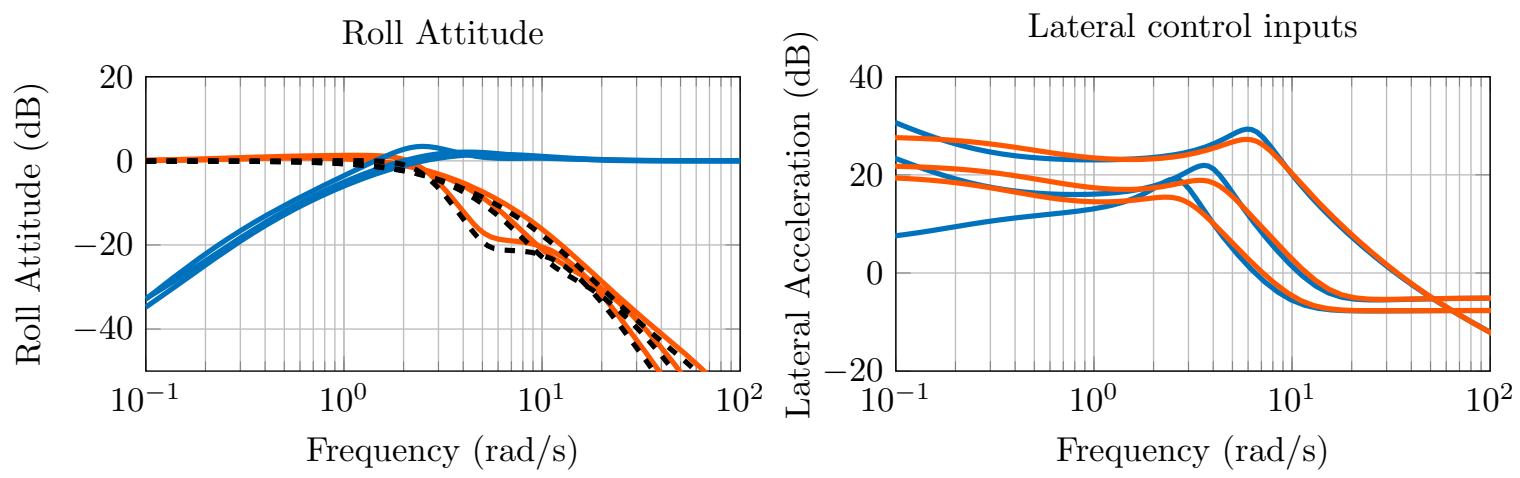

Figure 5. Left: Sensitivity ( -$)$, complimentary sensitivity $(-)$ and reference transmission (- - - ) for the roll attitude channel in the Bode Diagram (left). Right: Singular value plot from the lateral control inputs to lateral acceleration in the open loop (-) and closed loop ( -$)$. Both diagrams show lines for minimum, maximum and mean airspeed.
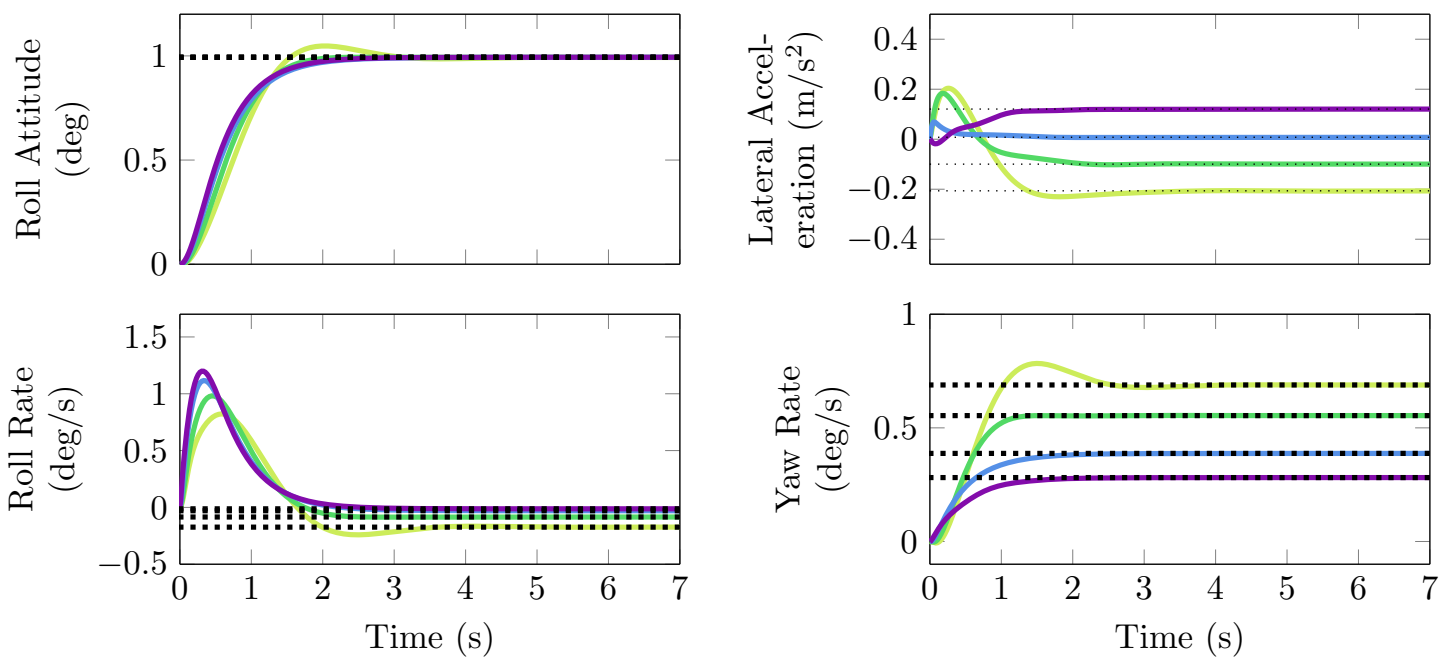

Figure 6. Linear closed loop step-responses in the lateral axis to a roll attitude command at $13 \mathrm{~m} / \mathrm{s}(-)$, $17 \mathrm{~m} / \mathrm{s}(\square), 25 \mathrm{~m} / \mathrm{s}(-), 35 \mathrm{~m} / \mathrm{s}$ ( $(-)$ ) airspeed.

adds damping and reduces the lateral acceleration. Note that this was not possible without the additional performance channel in the design.

\section{Anti Windup Augmentation}

In simulations using the linear aircraft model at low airspeeds, pitch axis responses showed occurrence of integrator windup inside the controller. As seen in Figure 10, when commanding a doublet in pitch attitude, the elevator deflection command is still at the saturation value, even though reference signal has already changed direction. To prevent this, anti-windup synthesis proposed in Ref. 18 is used.

\section{A. Theoretical background}

As shown in the block diagram Figure 7, which is used in modern anti-windup literature such as Ref. 19, windup inside the controller $K(s)$ is prevented through adding $y_{d}$ to the feedback. As a simple example, the choice of $M=I$ in (7) would in case of $G(s)$ being a linear plant simply result in the linear closed loop without saturation. To further enhance the performance (i.e. overshoots in the linear system could lead to $u_{m}<u_{\max }$ even though $y$ has not reached $r$ ), preconditioning with a second transfer function $M(s)$ can be used. Here, the plant dynamics can be replaced by faster poles. The result of the anti-windup synthesis shall be a stable transfer function from $\tilde{u}$ to $\left[\begin{array}{ll}u_{d} & y_{d}\end{array}\right]^{T}$. The plant model $G(s)$ and the closed loop over $K(s)$ 


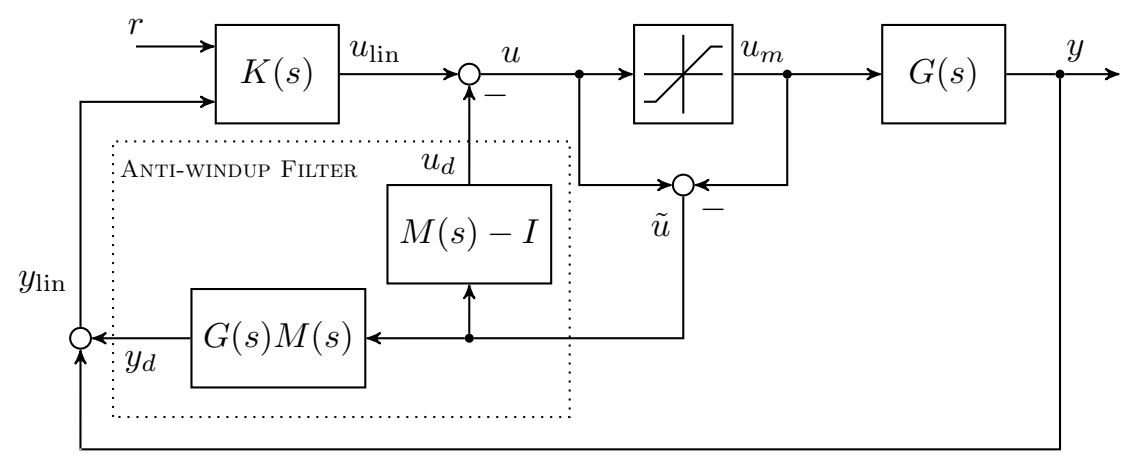

Figure 7. Anti-windup scheme with preconditioning.

need to be stable to allow this filter design. ${ }^{18}$ The anti-windup filter can be described as state-space model as in Ref. 18 by

$$
\left[\begin{array}{c}
\dot{\tilde{x}} \\
\hline u_{d} \\
y_{d}
\end{array}\right]=\left[\begin{array}{c|c}
A+B F & B \\
\hline F & 0 \\
C+D F & D
\end{array}\right]\left[\begin{array}{c}
\tilde{x} \\
\hline \tilde{u}
\end{array}\right] .
$$

The input $\tilde{u}$ to the system is the difference between the input and output of the actuator $\left(u-u_{m}\right)$. The matrices $A, B, C, D$ are the system matrices of the plant $G(s)$ and $F$ is the filter matrix to be designed. $F$ is calculated by solving a Riccati equation ${ }^{18}$

$$
\tilde{A}^{\prime} P+P \tilde{A}+P B R^{-1} B^{\prime} P+\tilde{Q}=0
$$

where

$$
\begin{aligned}
& \tilde{A}=A+B R^{-1} D^{\prime} C \\
& \tilde{Q}=C^{\prime}\left(I+D R^{-1} D^{\prime}\right) C \\
& R=\left(\gamma^{2} I-D^{\prime} D\right)>0 .
\end{aligned}
$$

and

$$
Z=\left(2 W-D^{\prime} D-\gamma^{-2} W^{2}\right)>0
$$

This leaves the free design parameters $\gamma$ and $W$ to be chosen, where $\gamma$ can be interpreted as gain which mixes between the fully linear response with $M=I$ and $M=0$. The latter would cancel the saturation of $u$ but would not prevent windup inside $K(s)$. W is selected as diagonal matrix with the same dimension as $D$ and is an additional design parameter which allows to define the poles of the anti-windup filter. With solving (3) and inserting $P$ into (6), the filter matrix is given by

$$
F=-\gamma^{2}\left(W^{-1}-\gamma^{-2} I\right) R^{-1}\left(B^{\prime} P+D^{\prime} C\right) .
$$

Inserting $F$ into (2) finally gives the anti-windup filter.

\section{B. Design application}

In the case of the pitch axis dynamics the feed-through matrix is equal zero, i.e. $D=0$. This simplifies the design problem to $\tilde{A}=A, \tilde{Q}=C^{\prime} C$ and $R=\gamma^{2} I$. Moreover, the parameters $\gamma$ and $W$ are scalars. As $\gamma$ shall be chosen smaller than $\|G(s)\|_{\infty}$ which is roughly 40 for $4 \times 4$ pitch dynamic model at design point of $\mathrm{V}=34 \mathrm{~m} / \mathrm{s}$, a value of $\gamma=30$ is selected to ensure a high $y_{d}$ compensation at the controller input. With the second condition $W<2 \gamma^{2}$ from Ref. 18, a maximum $W=900$ is theoretically possible. However, the maximum value for the anti-windup filter pole $W$, is practically constraint by the sampling time of $0.02 \mathrm{~s}$ of the flight control computer. This leads to an upper limit of $W=50$, which is finally chosen for the design. The functionality of the filter is verified in the next section. 


\section{Simulation based verification}

To provide a comprehensive verification of the control algorithms, the developed controllers are tested in a full non-linear model of the aircraft. The simulation model includes the aerodynamic lookup table data as described in Ref. 11, linear actuator models and sensor models with noise which were chosen in Refs. 7, 9.

\section{A. Controller validation over the full velocity range}

For proof of flight readiness, the controller is assessed in the closed loop simulation over the full velocity range. As maneuvers, pitch and roll attitude doublets are induced. Figure 8 depicts the results for a $10 \mathrm{deg}$ pitch attitude doublet. Compared to linear step responses only slight deviations are visible. These differences are mainly due to the additional lag induced by the sensor and actuator models. Simulations with altered aerodynamic parameters, inertias and disturbance inputs through simulated wind and gusts also show good results in terms of tracking and disturbance rejection. For the assessment of the LPV controller used to control the lateral dynamics, a $30 \mathrm{deg}$ roll attitude doublet is injected and shown in Figure 9 for different flying velocities. The LPV controller provides coherent roll attitude and roll rate responses at all velocities. Such a smooth behavior over all velocities could not be achieved with a constant $H_{\infty}$ controller design. The LPV controller also achieves improved tracking performance and less control effort in terms of maximum surface deflection compared to the $H_{\infty}$ controller. Moreover, oscillations in yaw rate and amplitudes in lateral acceleration have been minimized.
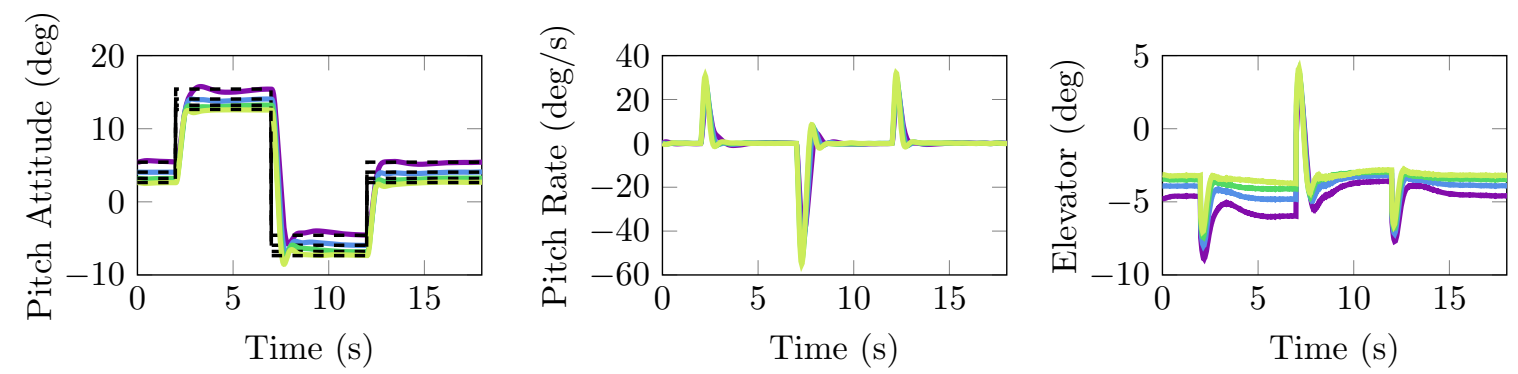

Figure 8. Longitudinal non-linear simulation response to a simulated pitch attitude doublet for $22 \mathrm{~m} / \mathrm{s}$ (—), $26 \mathrm{~m} / \mathrm{s}(\sqcup), 30 \mathrm{~m} / \mathrm{s}(\sqcup), 34 \mathrm{~m} / \mathrm{s}(-)$ airspeed .
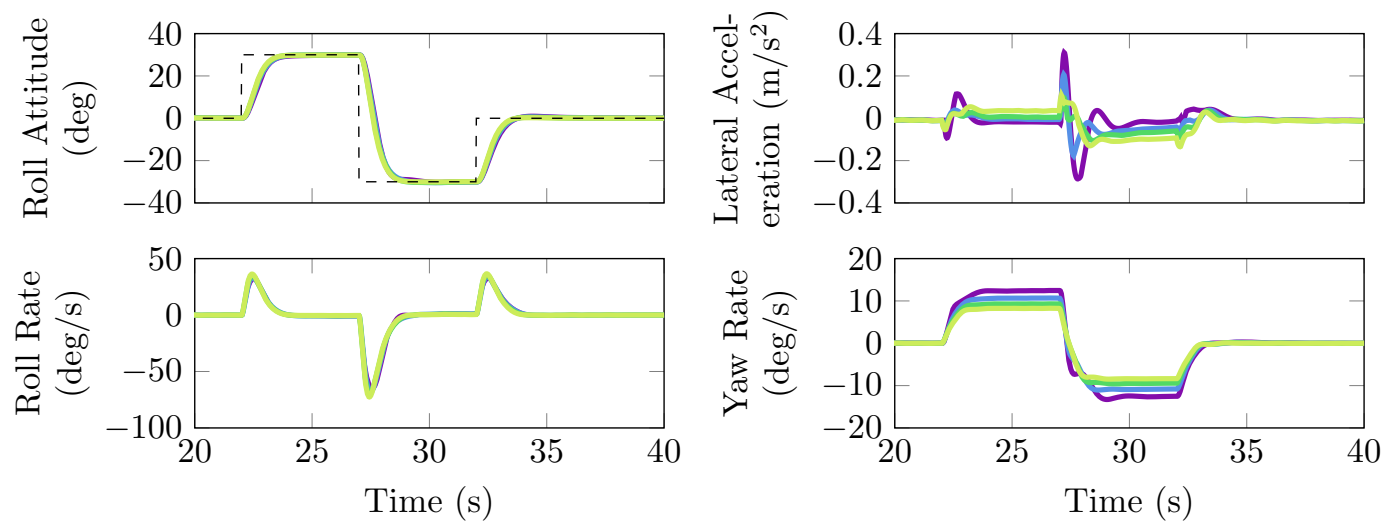

Figure 9. Lateral non-linear simulation responses to a roll attitude doublet for $22 \mathrm{~m} / \mathrm{s}(\longrightarrow), 26 \mathrm{~m} / \mathrm{s}(-)$, $30 \mathrm{~m} / \mathrm{s}(\longmapsto), 34 \mathrm{~m} / \mathrm{s}(-)$ airspeed.

\section{B. Anti-windup test}

In order to verify the functionality of the anti-windup compensator, a scenario with the previously presented pitch doublets is used. However, for this setup the upper output limit of the elevator command is set close 
to the trim value for horizontal flight. The simulation results are shown in Figure 10. The left diagrams shows the results without and the right diagrams the results with ant-windup compensation. The gray areas in all diagrams indicate that the actuator is at the saturation limit and wind-up can occur. The red area in case of no anti-windup compensation shows the effect of the wind-up, namely that the actuator remains at the saturation limit although the demanded signal has already changed direction. As soon as the green area starts, the actuator again is able to follow the commanded signal. In case of the anti-windup compensator active (right diagrams), the wind-up is completely avoided as the actuators is immediately able to follow the demanded position as soon as the command signal changes direction.
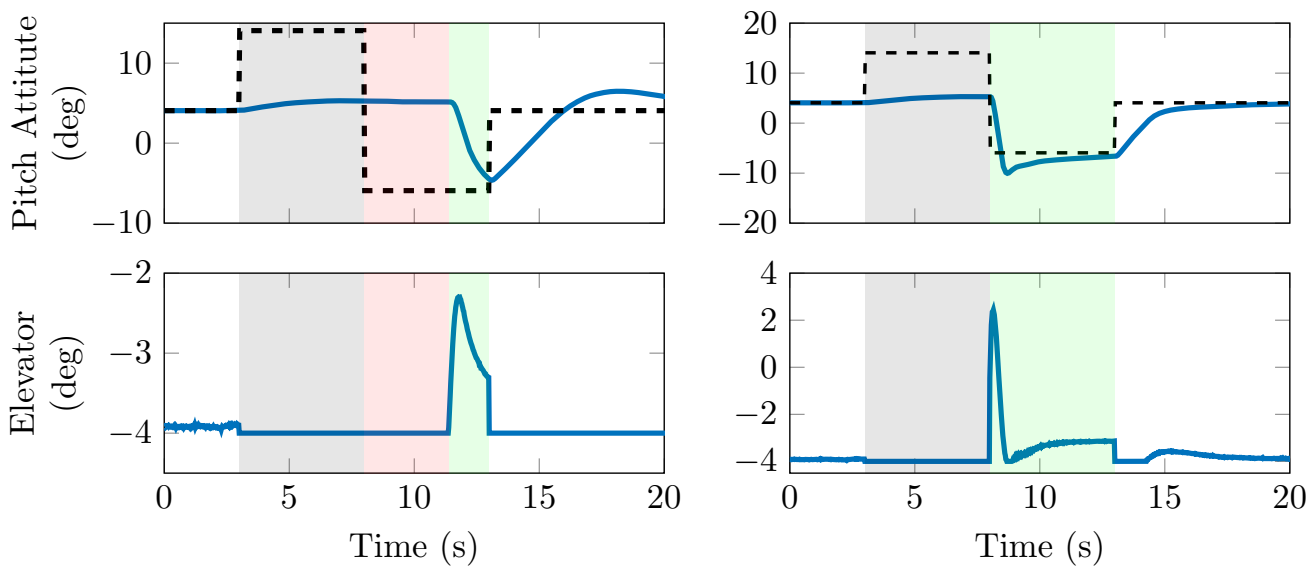

Figure 10. Non-linear simulation of pitch attitude doublet without (left) and with (right) anti-windup filter, using a reduced lower elevator limit at $-4 \mathrm{deg}$.

\section{Implementation aspects}

With the controllers validated there are a number of final steps before the software is flight ready, which are shortly described in this section. For implementation on the flight control system hardware, the controllers need to be discretized. This is done with a sample rate of $T_{s}=0.02 \mathrm{~s}$. For the discretization of the models, standard Matlab tools are used. Moreover, a reset-port is included which ensures a proper initialization of all discrete states for multiple on/off switching of the controller during flight. For the switch-on logic the following aspects are considered: when switching from manual to augmented mode, it is desired to fly wings leveled and with zero or small climb rate. This means, for roll axis it is sufficient to assume roll attitude of zero on switch-on and in augmented mode to map the aileron stick input from the remote control directly to a roll attitude command of $\pm 50 \mathrm{deg}$. To avoid open loop bumps during transfer, the last control surface position before switch-on is added onto the controller output.

For the longitudinal dynamics, it is important to consider the actual pitch attitude as well as the control surface deflection during switch-on. This helps to maintain a trimmed aircraft position and provides a smooth behavior during controller activation. To achieve this, when the controller is switched on the current stick position equals the current pitch attitude. Thus, during trimmed flight with constant flight-path angle, switching to augmented flight causes attitude-hold behavior as long as the stick position on the remote control is held constant. In controlled flight without pilot (pre-programmed doublet commands), the logic can be simplified as follows: the initial reference in pitch attitude is set to the current pitch attitude and the commanded elevator output is initialized at the last value before controller activation. The described logic has been implemented and validated in the nonlinear simulation model in various flight scenarios.

\section{Flight Testing}

Flight tests were executed at Aichach airfield near Augsburg, Germany. The tests were performed on two test days. Especially the second day featured strong wind conditions ( $\approx 13 \mathrm{kts})$ with heavy gusts, providing interesting conditions for the controller validation.

For execution of flight code and data capture, the Ultrastick 120 aircraft is equipped with a GOLDY 
flight computer. ${ }^{20}$ This includes besides the flight control computer unit also an internal measurement unit, air data sensors for static and dynamic pressure, a global positioning system, and data logging. During take off and landing, a safety pilot is in command and the aircraft can be flown manually via remote control.

When airborne, the safety pilot can switch the control laws on and off. The aircraft can then be flown in two different modes. In the first mode the flight control computer allows to execute pre-programmed maneuvers. The second mode enables the pilot to directly fly the controlled aircraft in an augmented mode. In this mode the pilot provides pitch and roll attitude commands via the stick on the remote control.

\section{A. Results}

In the first mode predefined pitch and poll doublets are automatically given as inputs to the FCS to ensure good comparability with previously obtained simulation results. Figure 11 shows the pitch axis results from four runs with $\pm 20 \mathrm{deg}$ pitch attitude doublets as inputs. In comparison the simulation results in Figure 8 , it can be observed that besides slightly higher tendencies for overshoots, the same tracking behavior is achieved with similar rise times and maximum pitch rates for different velocities and initial pitch attitudes. During runs with \pm 30 deg roll attitude doublets the aircraft was exposed to strong winds of $20-25 \%$ of the airspeed, with wind direction changing during the roll doublet maneuver from cross wind to headwind and back. However, the LPV controller manages to track the reference well despite these strong disturbances.
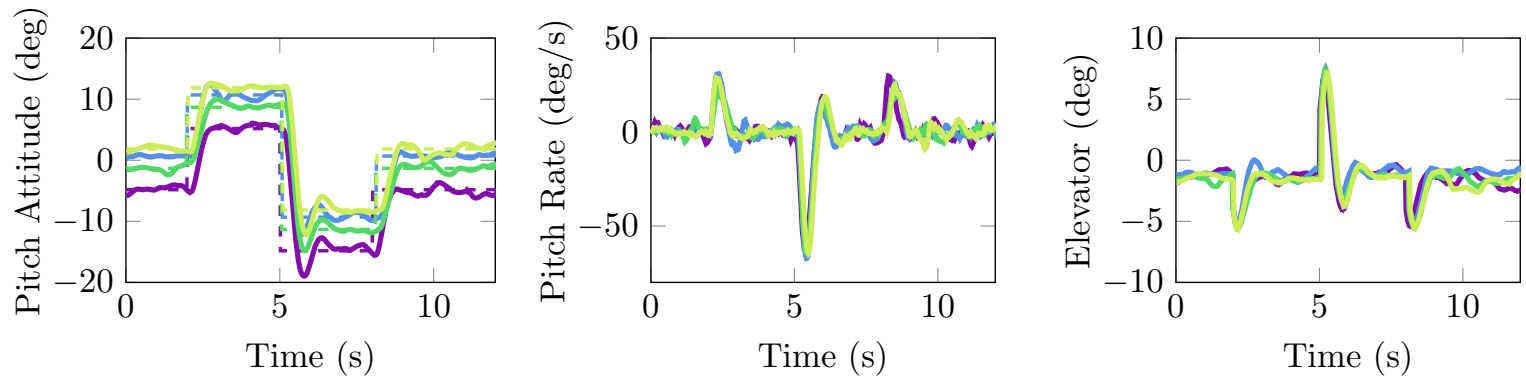

Figure 11. Longitudinal flight test results for a $10 \mathrm{deg}$ pitch attitude doublet at $27 \mathrm{~m} / \mathrm{s}(-,-), 30 \mathrm{~m} / \mathrm{s}$ $(\square), 32 \mathrm{~m} / \mathrm{s}(\square)$ airspeed.

In the second mode, the pilot remains in the loop and can command pitch and roll attitude directly via his remote control. During thee switch from manual mode to this augmented mode, the pilot tries to maintain wings level and zero or small climb rates. Hence, for the lateral axis it is possible to directly map the aileron stick input from the remote control to the roll attitude command of $\pm 50 \mathrm{deg}$ with the stick centered giving a roll command of zero. In the longitudinal axis, it is necessary to consider pitch attitude and control surface deflection in the control algorithm during switch-on in order to avoid sudden bumps and ensure a smooth switching. Therefore, the pitch attitude during switch-on references the current stick position, with an available range of $\pm 20 \mathrm{deg}$ in pitch attitude for the centered stick position. Maneuvers that have been executed in this mode include doublets similar to the predefined commands in the above described first mode, combinations of longitudinal and lateral commands, and step inputs with maximum pitch $(+20 \mathrm{deg})$ and maximum roll $(50 \mathrm{deg})$ command at the same time. These tests showed also good tracking and disturbance rejection behavior. Feedback of the pilot in command included that the handling qualities of the unmanned aircraft in augmented flight were excellent in terms of control effort and response time.

\section{B. Anti-windup flight test}

For validation of the anti-windup filter in flight, flight tests with decreased lower elevator saturation limits ( $-2.5 \mathrm{deg}$ for the first flight and $-1 \mathrm{deg}$ for the second flight) have been performed. To find these limits the elevator trim value for horizontal flight at $25 \mathrm{~m} / \mathrm{s}$ in simulation (-3 deg) set the basis for the selection. After one iteration in flight, the limit of $2.5 \mathrm{deg}$ was found to cause a satisfactory triggering of the integrator windup. For the test with the anti-windup compensator the even tighter limit of $-1 \mathrm{deg}$ was selected. Initial tests also showed that the triggering of the integrator windup in flight with pure pitch up commands (and zero roll attitude) was hardly possible as during these pure pitch ups with pitch attitudes of $30 \mathrm{deg}$ and more 

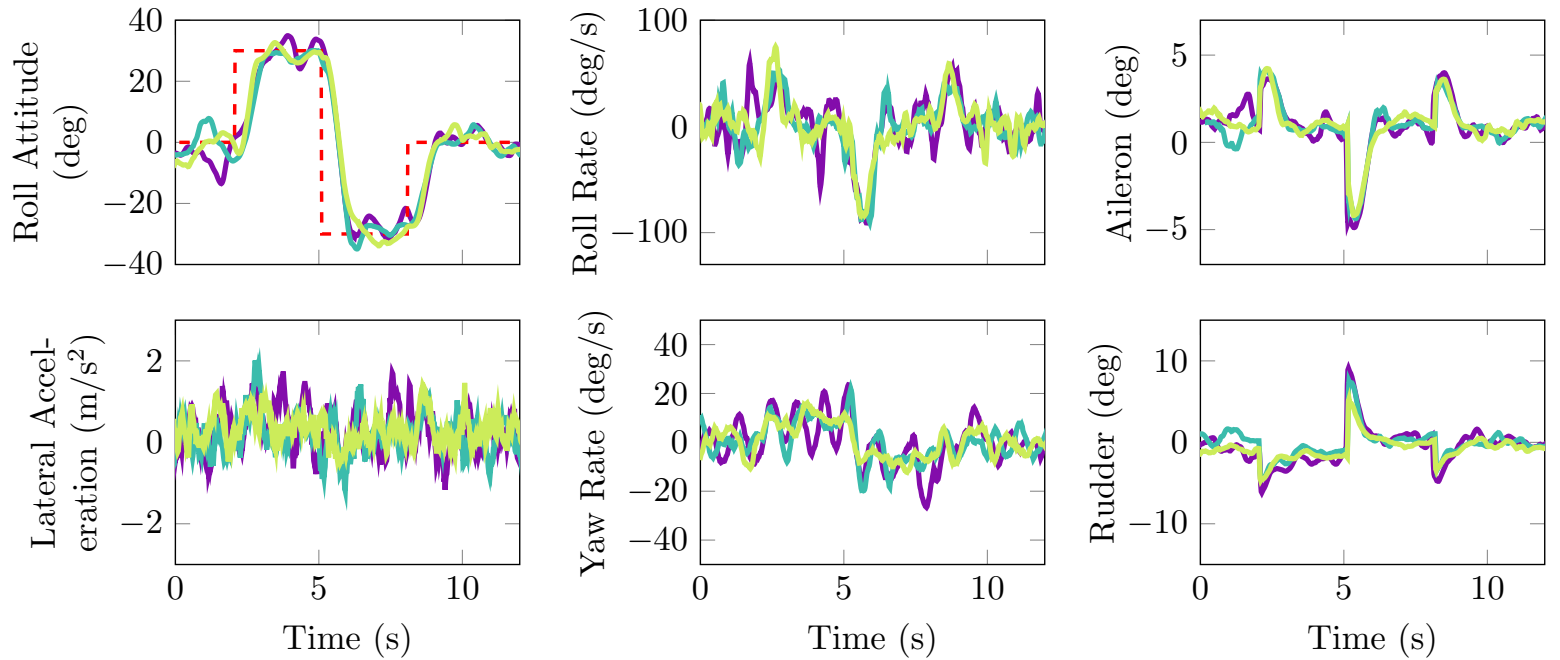

Figure 12. Lateral flight test results to a $30 \mathrm{deg}$ roll attitude doublet at $27 \mathrm{~m} / \mathrm{s}(-), 29 \mathrm{~m} / \mathrm{s}(-), 31 \mathrm{~m} / \mathrm{s}$ $(-)$ airspeed.

over longer times the risk of damage to the engine and the batteries due too overpowering these components was too high. Thus, turns in which roll and pitch attitude were increased at the same time were performed to trigger the windup. The diagrams in Figure 13 show the comparison between a pitch up maneuver flown with and without anti-windup filter.
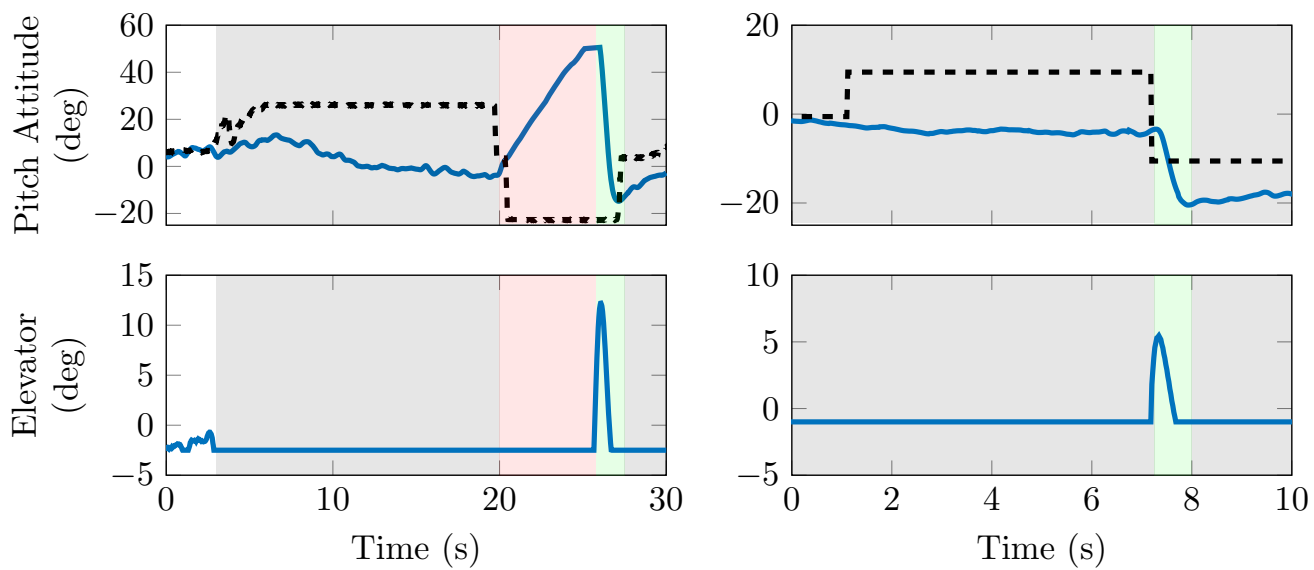

Figure 13. Flight-test results without (left) and with (right) anti-windup filter for reduced elevator limits.

In both cases the reference pitch-up commands cannot be tracked due to the artificially lowered elevator limit. In the left case without anti-windup compensation, the turn is ended and at the same time $(\approx 20 \mathrm{~s}) \mathrm{a}$ pitch down command is given by the pilot. It is clearly visible, that the elevator remains in the saturation for more than $5 \mathrm{~s}$ before the aircraft is able to pitch down again. During this time, due to returning to wings leveled flight, the aircraft starts even pitching up and reaches 50 deg in pitch attitude. This unwanted pitchups are avoided by adding the anti-windup compensator, which was done for the tests in the right diagrams. Again, the elevator position is in the saturation and the pitch attitude cannot follow the demanded input signal. However, with anti-windup compensator active, the control surface reacts immediately as soon as the reference signal changes to a negative value, confirming the correct design and functionality of the designed anti-windup compensator. 


\section{Conclusions}

In this work a LPV / $H_{\infty}$ based flight control system featuring an advanced anti-windup method has been developed for a small unmanned aircraft. The obtained flight controller has been validated through extensive simulations and a flight test campaign. The results show the successful implementation of the controller design tool-chain, including controller design itself, (non-linear) simulation based verification and in-flight controller validation on an Ultrastick 120 UAV. In detail, a flight control algorithm has been obtained, which meets the previously set design goals in tracking behavior, robustness against model uncertainties and disturbance rejection. Additionally, the applicability and effectiveness of an advanced anti-windup strategy based on model based preconditioning has been proven in simulations as well as in flight tests.

\section{Acknowledgment}

The authors would like to thank the DLR flight test team, in particular Richard Kuchar, Daniel Milz and Davey Kroezen, who supported the preparation and performance of the flight test campaign. In addition we thank our pilot, Bernd Dörfler from Rödel Aircraft-Systems, who executed all flights and provided valuable feedback.

\section{References} 2011.

${ }^{1}$ Brockhaus, R., Alles, W., and Luckner, R., Flugregelung, Springer-Verlag Berlin Heidelberg, Berlin Heidelberg, 3rd ed.,

${ }^{2}$ Hanel, M. and Well, K., Controller Design for a Flexible Aircraft, Vol. 48, Springer, Boston, MA, 2004, pp. 155-180.

${ }^{3}$ Theis, J., Pfifer, H., and Seiler, P., "Robust Control Design for Active Flutter Suppression," Proc. of AIAA Guidance, Navigation, and Control Conference, San Diego, CA, 2016, pp. 1-13.

${ }^{4}$ Pusch, M., "Aeroelastic Mode Control using $H_{2}$-optimal Blends for Inputs and Outputs," Proc. of AIAA Guidance, Navigation, and Control Conference, Kissimmee, FL, 2018, pp. 1-12.

${ }^{5}$ Gaulocher, S., Roos, C., and Cumer, C., "Aircraft Load Alleviation During Maneuvers Using Optimal Control Surface Combinations," Journal of Guidance, Control, and Dynamics, Vol. 30, 2007, pp. 591-600.

${ }^{6}$ Ossmann, D. and Poussot-Vassal, C., "Minimal order disturbance estimator design for aircraft load alleviation control," IEEE Conference on Control Technology and Applications, Vol. 2, 2018.

${ }^{7}$ Dorobantu, A., Johnson, W., Lie, F. A., Taylor, B., Murch, A., Paw, Y., Gebre-Egziabher, D., and Balas, G., "An Airborne Experimental Test Platform: From Theory to Flight," Proc. of American Control Conference, Wahsington, DC, 2013, pp. 659-673.

${ }^{8}$ Sartori, D., Quagliotti, F., Rutherford, M., and Valavanis, K., "Implementation and testing of a backstepping controller autopilot for fixed-wing UAVs," Journal of Intelligent $\mathcal{E}$ Robotic Systems, Vol. 76, 2014, pp. 505-525.

${ }^{9}$ van Ekeren, W., Incremental Nonlinear FlightControl for Fixed-Wing Aircraft, Master's thesis, TU Delft, 2016.

${ }^{10}$ Owens, B., "Development of a low-cost sub-scale aircraft for flight research: The FASER project," Proc. of 25th AIAA Aerodynamic Measurement Technology and Ground Testing Conference, San Francisco, California, 2006, pp. 1-11.

${ }^{11}$ Hoe, G., Owens, D., Bruce, D., and Denham, C., "Forced Oscillation Wind Tunnel Testing for FASER Flight Research Aircraft," Proc. of AIAA Atmospheric Flight Mechanics Conference, American Institute of Aeronautics and Astronautics, Reston, Virigina, 2012, pp. 1-11.

${ }^{12}$ Skogestad, S. and Postlethwaite, I., Multivariable feedback control: Analysis and design, Wiley, Chichester, 2nd ed., 2005.

${ }^{13}$ Theis, J., Pfifer, H., Balas, G., and Werner, H., "Integrated flight control design for a large flexible aircraft," Proc. of American Control Conference, 2015, pp. 3830-3835.

${ }^{14}$ Theis, J., Ossmann, D., and Pfifer, H., "Robust Autopilot Design for Crosswind Landing," Proc. of IFAC World Congress, Toulouse, France, 2017.

${ }^{15}$ Theis, J., Ossmann, D., Thielecke, F., and Pfifer, H., "Robust autopilot design for landing a large civil aircraft in crosswind," Control Engineering Practice, Vol. 76, 2018, pp. $54-64$.

${ }^{16} \mathrm{Wu}, \mathrm{F} .$, Control of Linear Parameter Varying Systems, Ph.D. thesis, University of Calicfornia, University of California, Berkeley, 1995.

${ }^{17}$ Hjartarson, A., Seiler, P., and Packard, A., "LPVTools: A Toolbox for Modeling, Analysis, and Synthesis of Parameter Varying Control Systems," IFAC-PapersOnLine, Vol. 48, No. 26, 2015, pp. 139-145.

${ }^{18}$ Sofrony, J., Turner, M. C., and Postlethwaite, I., "Anti-windup synthesis using Riccati equations," International Journal of Control, Vol. 80, No. 1, 2007, pp. 112-128.

${ }^{19}$ Zaccarian, L. and Teel, A. R., Modern anti-windup synthesis: Control augmentation for actuator saturation, Princeton series in applied mathematics, Princeton Univ. Press, Princeton NJ, 2011.

${ }^{20}$ Johnson, W., Goldy Flight Control System v1.0, 2014. 